\title{
Accumbens Mechanisms for Cued Sucrose Seeking
}

\author{
Ana-Clara Bobadilla*,', Constanza Garcia-Keller', Jasper A Heinsbroek', Michael D Scofield', \\ Victoria Chareunsouk', Cara Monforton' and Peter W Kalivas*,' \\ 'Department of Neurosciences, Medical University of South Carolina, Charleston, SC, USA
}

\begin{abstract}
Many studies support a perspective that addictive drugs usurp brain circuits used by natural rewards, especially for the dopaminedependent reinforcing qualities of both drugs and natural rewards. Reinstated drug seeking in animal models of relapse relies on glutamate spillover from cortical terminals synapsing in the nucleus accumbens core (NAcore) to stimulate metabotropic glutamate receptor5 (mGluR5) on neuronal nitric oxide synthase (nNOS) interneurons. Contrasting the release of dopamine that is shared by sucrose and drugs of abuse, reinstated sucrose seeking does not induce glutamate spillover. We hypothesized that pharmacologically promoting glutamate spillover in the NAcore would mimic cocaine-induced adaptations and potentiate cued reinstatement of sucrose seeking. Inducing glutamate spillover by blocking astroglial glutamate transporters (GLT-I) had no effect on reinstated sucrose seeking. However, glutamate release probability is negatively regulated by presynaptic mGluR2/3, and sucrose reinstatement was potentiated following mGluR2/3 blockade. Potentiated sucrose reinstatement by mGluR2/3 blockade was reversed by antagonizing mGluR5, but reinstated sucrose seeking in the absence of mGluR2/3 blockade was not affected by blocking mGluR5. In cocaine-trained rodents mGluR5 stimulation reinstates drug seeking by activating $\mathrm{nNOS}$, but activating mGluR5 did not promote reinstated sucrose seeking, nor was potentiated reinstatement after mGluR2/3 blockade reduced by blocking $n N O S$. However, chemogenetic activation of nNOS interneurons in the NAcore reinstated sucrose seeking. These data indicate that dysregulated presynaptic mGluR2/3 signaling is a possible site of shared signaling in drug seeking and potentiated reinstated sucrose seeking, but that downregulated glutamate transport and subsequent activation of $\mathrm{nNOS}$ by synaptic glutamate spillover is not shared.

Neuropsychopharmacology (2017) 42, 2377-2386; doi:I0.1038/npp.2017.153; published online 16 August 2017
\end{abstract}

\section{INTRODUCTION}

A widespread perspective in the field of drug addiction is that drugs of abuse 'hijack' physiological brain reward circuits that mediate responding to natural rewards such as sex and food (Bechara, 2005; Schultz, 2011). This perspective is supported by shared behavioral characteristics between seeking of natural rewards and substance use disorders (Ahmed et al, 2013; Oginsky et al, 2016b; Schreiber et al, 2013), and has proven useful for understanding the importance of mesocorticolimbic dopamine circuitry in the reinforcing properties of both addictive drugs and biological rewards. Addictive drugs and natural rewards also broadly impact brain circuits in which the dopamine projections are embedded. For example, clinical imaging studies show that cue-induced motivation to seek natural rewards or addictive drugs activates prefrontal cortex to nucleus accumbens circuitry (Tomasi and Volkow, 2013).

Contrasting general evidence of shared dopamine circuit involvement, addictive drugs produce enduring changes at glutamatergic synapses in the nucleus accumbens that are

\footnotetext{
* Correspondence: Dr A-C Bobadilla or Dr PW Kalivas, Medical University of South Carolina, Department of Neurosciences, 173 Ashley Avenue, BSB 403- MSC 5I0, Charleston, SC 29425, USA, Tel: 843876 2246, Fax: 843792 4423, E-mail: bobadilla@musc.edu or kalivasp@musc.edu Received 21 December 2016; revised 10 July 2017; accepted II July 2017; accepted article preview online 20 July 2017
}

required for cue-induced drug-seeking behavior and may not be shared with cue-induced sucrose seeking. For example, cue-induced spillover of synaptic glutamate occurs during reinstated seeking of many addictive drugs, including cocaine, alcohol, nicotine, heroin, and methamphetamine, but not during sucrose seeking (Luscher and Malenka, 2011; Mulholland et al, 2016; Scofield et al, 2016a; Wolf, 2010), although glutamate spillover was shown in obesity prone rats (Brown et al, 2015). Spillover of synaptic glutamate during drug seeking is facilitated by two enduring adaptations elicited in the core subcompartment of the nucleus accumbens core (NAcore) by most addictive drugs. Astroglial glutamate transport by GLT-1 is reduced causing glutamate to accumulate in the extracellular space (Danbolt, 2001; Rao and Sari, 2012; Scofield et al, 2016a), and presynaptic glutamate release probability is enhanced due to decreased regulation by metabotropic glutamate autoreceptors (mGluR2/3) (Mulholland et al, 2016). Supporting a shared mechanism between drug and natural reward, stimulating mGluR2/3 to decrease synaptic release probability reduces both reinstated sucrose and drug seeking (Baptista et al, 2004; Bossert et al, 2006; Peters and Kalivas, 2006). However, drugs that restore GLT-1 function (eg ceftriaxone or $\mathrm{N}$-acetylcysteine) reduce cocaine, heroin, alcohol, and nicotine seeking but do not impact reinstated sucrose seeking (Scofield et al, 2016a). 
Recently we demonstrated that cue-induced glutamate spillover during cocaine seeking stimulates mGluR5 on neuronal nitric oxide synthase (nNOS) interneurons in NAcore (Smith et al, 2017). Correspondingly, the mGluR5dependent production of nitric oxide induces cocaineseeking behavior in absence of cues. It is unknown if the NAcore intrinsic circuit necessary for reinstated cocaine that involves glutamate spillover to initiate mGluR5-dependent stimulation of nitric oxide synthesis plays a role in reinstated sucrose seeking.

Here, we examined the involvement of altered glutamate homeostasis in cued sucrose seeking by determining if pharmacologically inducing glutamate spillover using either an mGluR2/3 antagonist or GLT1 inhibitor potentiated reinstated sucrose seeking. We also examined if the NAcore intrinsic nNOS circuit is involved in sucrose seeking. We found that blockade of mGluR2/3, but not GLT1, potentiated reinstated sucrose seeking in an mGluR5-dependent manner, and that mGluR2/3 potentiation did not require nNOS activity. However, when nNOS activity was chemogenetically increased, cued sucrose seeking was potentiated.

\section{MATERIALS AND METHODS}

\section{Animals and Surgery}

Male Sprague Dawley rats (200-250 g, Charles River Laboratories) were housed individually in a temperaturecontrolled environment on a 12-h reverse light cycle. After 1 week of acclimation, animals were anesthetized with ketamine $(100 \mathrm{mg} / \mathrm{kg})$ and xylazine $(7 \mathrm{mg} / \mathrm{kg})$, together with ketorolac analgesic $(0.28-0.32 \mathrm{mg} / \mathrm{kg})$ and prophylactic antibiotic (Cefazolin, $200 \mathrm{mg} / \mathrm{ml}$, subcutaneous; West-Ward Pharmaceuticals, NJ). Bilateral 26 gauge cannulas (PlasticsOne, VA) were inserted targeting the NAcore (+1.5 A/P, $+1.7 \mathrm{M} / \mathrm{L},-5.5 \mathrm{D} / \mathrm{V})$, secured in place with dental cement and maintained open with $0.009^{\prime \prime} \times 30^{\prime \prime}$ internal cannulas. Rats had 1 week to recover from surgery before starting behavioral training.

Male NOS1-Cre transgenic mice ( $25 \mathrm{~g}$; Jackson Labs \#017526 B6.129-Nos1tml(cre_Mgmj/J) were individually housed on a 12-h reverse light cycle. After at least 1 week of acclimation, mice (25-30 g) were anesthetized with isofluorane (induction $3-5 \% \mathrm{v} / \mathrm{v}$, maintenance $1-2 \% \mathrm{v} / \mathrm{v}$ ) and $\mathrm{AAV}$ viral vector (AAV2.hSyn.DIO.hM3D.mCherry; titer $4.6 \times 10^{12}$, $300 \mathrm{nl}$ per side, $100 \mathrm{nl}$ per min, 5-10 min diffusion; University of North Carolina Vector Core, Chapel Hill, NC) was infused in the nucleus accumbens (AP: +1.5, ML: $\pm 1.2, \mathrm{DV}:-4.4$ ). Animals were given NSAID (carprofen $5 \mathrm{mg} / \mathrm{kg}$ s.c.) and antibiotics (Cefazolin $200 \mathrm{mg} / \mathrm{kg}$ i.v.) and were allowed to recover from surgery for at least 1 week before starting behavioral training.

\section{Reagents}

Drugs microinjected in the NAcore included mGluR5 agonist (RS)-2-chloro-5-hydroxyphenylglycine $\quad((\mathrm{CHPG})=30 \mu \mathrm{g} / \mu \mathrm{l}$, Tocris), non-selective glutamate reuptake antagonist threo- $\beta$ benzyloxyaspartate $((\mathrm{TBOA})=0.5 \mathrm{nmol} / \mu \mathrm{l}$, Tocris $), \mathrm{mGluR2}-$ 13 antagonist LY $341495(4 \mu \mathrm{g} / \mu \mathrm{l}$, Tocris), mGluR5 negative allosteric modulator (NAM) 3-((2-methyl-4-thiazolyl)ethynyl) pyridine $((\mathrm{MTEP})=2 \mu \mathrm{g} / \mu \mathrm{l}$, Tocris $)$ and nNOS inhibitor $N$ - propyl L-arginine hydrochloride $((\mathrm{NPLA})=0.2 \mathrm{nmol} / \mu \mathrm{l}$, Tocris). Clozapine $\mathrm{N}$-oxide $((\mathrm{CNO})=0.3 / 1 / 3 \mathrm{mg} / \mathrm{kg}$, National Institute of Drug Abuse drug supply program) was administrated i.p. All reagents were diluted in saline, except $\mathrm{CNO}$, which was dissolved in $0.5 \%$ DMSO and saline.

\section{Sucrose Self-Administration Procedures}

Self-administration was performed on an FR1 (10 days) or FR1/FR3/FR5 (4/3/3 days) reinforcement schedule (2 h per day, standard rat or mouse modular test chambers (Med Associates, Fairfax, VT) during which active lever presses resulted in delivery of a sucrose pellet ( $45 \mathrm{mg}$ for rats, $15 \mathrm{mg}$ for mice, Bio-Serv, Flemington, NJ) paired with a compound (light and tone, $78 \mathrm{~dB}, 4.5 \mathrm{kHz}$ ) cue. Following a minimum of 10 days of 10 pellets or more, animals entered extinction training, during which time lever presses had no consequences. Extinction criterion was reached and animals were tested when the average of active lever presses during the 3 last days of extinction was equal or less than $30 \%$ of the average of the last 3 days of self-administration, which usually happened after 10-12 days of extinction training. Both rats and mice reached criteria approximately after the same amount of sessions, keeping the length of learning between species similar (10 days in sucrose self-administration, 10-12 days of extinction training). Animals were maintained on food restriction ( $22 \mathrm{~g}$ chow per day for rats, $4 \mathrm{~g}$ for mice) throughout behavioral testing. Behavioral sessions were carried out between 1200 and $1600 \mathrm{~h}$.

\section{Reinstatement, Microinjections, and Histology}

In rats, during the $2 \mathrm{~h}$ reinstatement tests sucrose seeking was induced by contingent exposure to the cues previously paired with sucrose, but without sucrose delivery. Rats were microinjected $2 \mathrm{~mm}$ below the cannula base using 33-gauge microinjectors at $0.25 \mu \mathrm{l} / \mathrm{min}$ for a total volume of $0.5 \mu \mathrm{l}$ per hemisphere. Microinjectors were left in place to allow diffusion for $2 \mathrm{~min}$. Animals were returned to home cage for 10 additional minutes before starting reinstatement sessions to allow recovery from the microinjection procedure. For all experiments, rats tested within-subjects and counter-balanced up to 3 cued-reinstatements.

In mice, reinstated sucrose seeking was tested in absence of cues previously paired with sucrose ( $2 \mathrm{~h}$ extinction test) and in the presence of cues ( $2 \mathrm{~h}$ cued-reinstatement). Mice received an injection of $\mathrm{VEH}$ or $\mathrm{CNO}$ i.p. $30 \mathrm{~min}$ before testing, in a within-subjects counter-balanced fashion. At the end of all experiments, rats and mice were transcardially perfused with $0.9 \%$ saline followed by a $10 \%$ phosphatebuffered formalin solution. Brains were sliced at $100 \mu \mathrm{m}$ on a vibratome after which cannula placements or virus expression and localization of infection were verified by visualizing mCherry expression using an epifluorescent microscope.

\section{Immunohistochemistry}

The protocol for immunohistochemistry has been described elsewhere (Smith et al, 2017). Briefly, mice were deeply anesthetized with pentobarbital, perfused with PBS followed by $4 \%$ paraformaldehyde, and brains were post-fixed for 
$24 \mathrm{~h}$. Using a vibratome, brains were sliced in $100 \mu \mathrm{m}$ sections. Free-floating sections were rinsed in PBS-Triton $(0.1 \%)$ and incubated in normal goat serum and primary antibodies (Anti-Cre Recombinase, Millipore \#MAB3120 Clone 2D8, 1:1000; Anti-nNOS, Millipore \#AB5380, 1:1000) overnight at $4{ }^{\circ} \mathrm{C}$. After multiple PBS-Triton rinses, sections were incubated in Alexa-Fluor-conjugated secondary antibodies (Life Technologies, 1:1000). Images were taken on a confocal microscope (Leica) using a $\times 63$ objective and deconvolved using AutoQuant X2 (MediaCybernetics).

\section{Locomotor Activity}

The effect of reagents was tested on spontaneous locomotion by employing the same treatment injection procedures used before reinstatement testing (microinjections for rats, i.p. injections for mice) followed by a $1 \mathrm{~h}$ open field session for rats using Digiscan Animal Activity Monitor system (Omnitech Electronics Model RXYZCM, Columbus, OH) and $2 \mathrm{~h}$ locomotor session for mice in a designated locomotor apparatus (Med Associates, Fairfax, VT).

\section{Statistics}

When two groups were compared, the data were statistically analyzed using a two-tailed unpaired Student's $t$-test. For comparing multiple measurements in the same experiment, the data were analyzed using 1-, 2- or 3-way ANOVAs as indicated in the text describing each experiment. Sidak's post-hoc test was applied for multiple comparisons and $p<0.05$ was considered statistically significant. All statistical tests are conducted using the Prism (Graphpad, La Jolla, CA) software package.

\section{RESULTS}

\section{Rat Sucrose Self-Administration and Cue-Induced Reinstatement of Sucrose Seeking}

Using a fixed ratio 1 (FR1), animals readily self-administered sucrose and discriminated between the active and the inactive levers (Figure 1a). We also used an ascending FR1/ FR3/FR5 schedule of reinforcement in some experiments to engender higher levels of active lever responding (Figure 1b), which correspondingly amplified cue-induced reinstatement (Figure $1 \mathrm{c}$ and d). Both self-administration protocols resulted in significant cue-induced reinstatement on the active lever compared to extinction active lever and reinstatement inactive lever pressing (Figure $1 \mathrm{c}$ and $\mathrm{d}$ ). Figure $1 \mathrm{a}, \mathrm{b}$ and e illustrates behavioral data and histology for all rats used in the subsequent reinstatement studies. Figure 1c and d pools the reinstatement for all vehicle-pretreated rats after FR1 or FR1/FR3/FR5 schedules of reinforcement that were included as control groups in subsequent experiments.

\section{Blocking GLT-1 or Stimulating mGluR5 Signaling Did Not Increase Reinstated Sucrose Seeking in Rats}

Pharmacologically blocking GLT-1 with TBOA increases the extracellular concentration of glutamate in the nucleus accumbens (Baker et al, 2002; Griffin et al, 2014; Oldenziel et al, 2006). We microinjected TBOA $(0.25 \mathrm{nmol} / 0.5 \mu \mathrm{l}$ (Griffin et al, 2014)) into the NAcore $10 \mathrm{~min}$ prior to initiating the reinstatement session (Figure 1e for histology). TBOA had no effect compared to vehicle on reinstated sucrose seeking in rats trained on an FR1 schedule of reinforcement (Figure 2a). Using the FR1/FR3/FR5 schedule of reinforcement, TBOA showed a non-significant trend to reduce cued reinstatement since a 3 -way ANOVA revealed that active lever pressing was elevated over inactive pressing but was not significantly different from active lever pressing during extinction training (Figure 2b). However, two-way ANOVA analysis of active lever pressing shows no significant effect of treatment between any groups (ie vehicle vs TBOA). TBOA did not alter inactive lever pressing relative to vehicle-microinjected rats after training with either schedule of reinforcement (Figure $2 \mathrm{a}$ and $\mathrm{b}$ ).

Given the capacity of the mGluR5 agonist CHPG to induce cocaine seeking in cocaine-extinguished animals and to potentiate cue-induced reinstatement of cocaine seeking when microinjected in the NAcore (Smith et al, 2017; Wang et al, 2013), we examined in rats the effect of direct pharmacological activation of Gq-coupled mGluR5. CHPG microinjections into the NAcore $(30 \mu \mathrm{g} / \mathrm{side})$ did not alter sucrose seeking in extinguished (Figure $2 c$ ) or cue reinstated rats (Figure 2d). Both the extinction test and cuedreinstatement were performed within-subjects.

\section{Blockade of mGluR2/3 Potentiated Rat Reinstated Sucrose Seeking via mGluR5 Stimulation}

Glutamate release probability is negatively regulated by presynaptic mGluR2/3, and blocking mGluR2/3 promotes synaptic glutamate spillover (Moussawi and Kalivas, 2010). We therefore microinjected a previously published dose of the mGluR2/3 antagonist, LY 341495, into the NAcore prior to reinstatement in order to increase glutamate release probability (Moussawi et al, 2011). LY 341495 potentiated cue-induced sucrose seeking (Figure 3a), which appeared to be specific to seeking behavior since there was no increase in inactive lever pressing (Figure 3a) or locomotion in an open field (Figure 3b).

Glutamate spillover during reinstated cocaine seeking stimulates mGluR5 (Smith et al, 2017), and mGluR5 antagonists inhibit reinstated drug seeking (Olive, 2009; Scofield et al, 2016a). Consistent with a previous study, we found that inhibiting mGluR5 did not reduce reinstated sucrose seeking (Sinclair et al, 2012). However, microinjecting the mGluR5-negative allosteric modulator MTEP with LY 341495 into the NAcore prior to cue-reinstated sucrose seeking prevented the LY 341495-induced potentiation of sucrose seeking (Figure 3a). MTEP-induced blockade was independent from overall locomotion, since MTEP microinjections in the NAcore had no effect on open field locomotion (Figure $3 \mathrm{~b}$ ).

The blockade by mGluR5 blockade indicated that LY 341495-induced increase in glutamate spillover was accessing mGluR5 outside of the synapse. mGluR5 is expressed widely on NAcore neurons (Luscher and Huber, 2009), but cue-reinstated cocaine seeking depends specifically on mGluR5 stimulation of nitric oxide synthesis in the $\sim 1 \%$ of NAcore neurons that express nNOS (Smith et al, 2017). To investigate a role for mGluR5 activation of nNOS we microinjected a selective nNOS inhibitor, NPLA ( $1 \mathrm{nmol}$; a behaviorally effective dose at inhibiting cued 

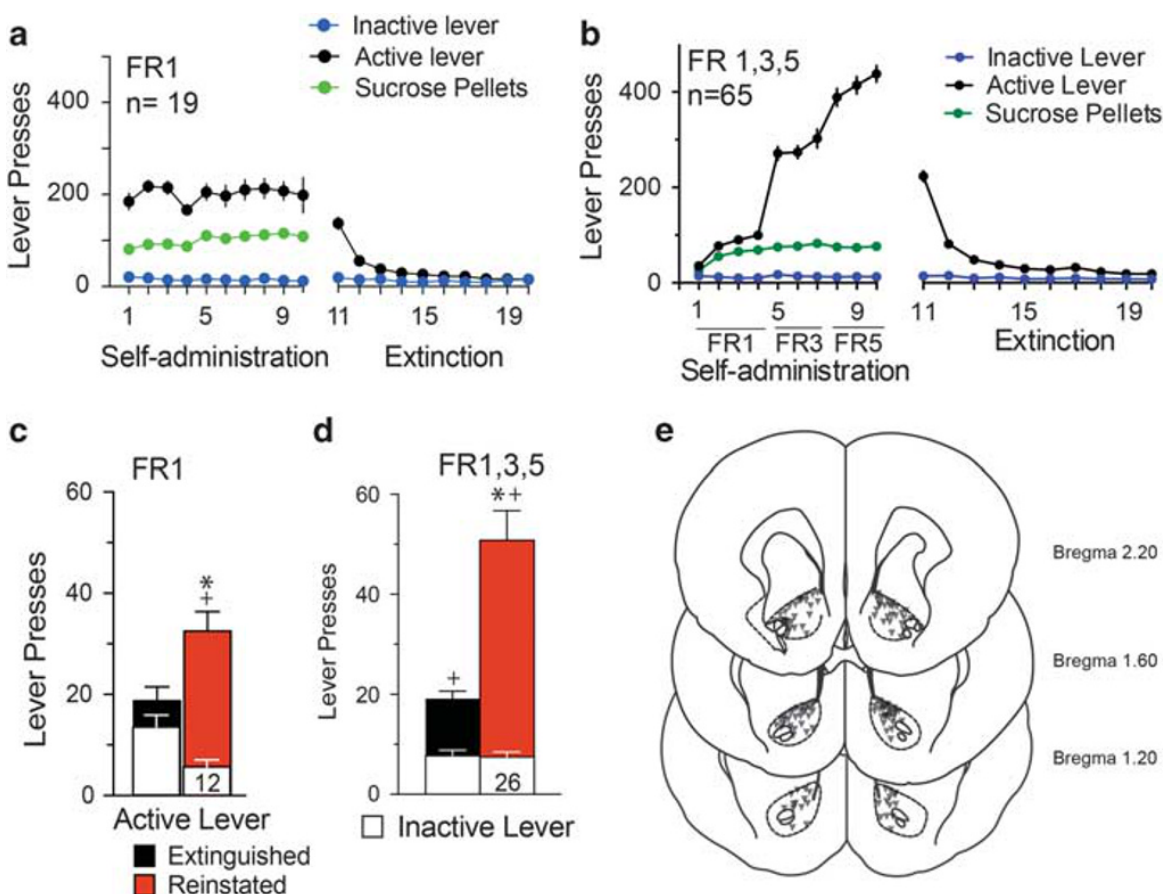

Figure I Rat sucrose self-administration, extinction training, and cue-induced reinstatement. (a) Sucrose self-administration with fixed ratio I (FRI) and extinction training, $n=19$. (b) Sucrose self-administration with increasing schedule of reinforcement FRI/FR3/FR5 and extinction training, $n=65$. (c) Cuedreinstatement after an FRI schedule and extinction training corresponding to the $n=12$ vehicle microinjections. Two-way ANOVA active/inactive lever $F_{(I, 22)}=25.21, p<0.001$, extinction/reinstatement $F_{(I, 22)}=1.73, p=0.202$, interaction $\left(F_{(1,22)}=22.62, p<0.00 I\right)$. (d) Cued-reinstatement after an FRI/FR3/ FR5 schedule sucrose self-administration and extinction training corresponding to $n=26$ vehicle microinjections. Two-way ANOVA active/inactive lever $F_{(I, 25)}=65.08, p<0.001$, extinction/reinstatement $\left(F_{(1.25)}=32.63, p<0.001\right.$, interaction $\left.F_{(1.25)}=37.65, p<0.001\right)$. * $p<0.05$ comparing extinction to reinstatement active lever pressing using a Sidak's multiple comparisons post-hoc test. ${ }^{+} p<0.05$ comparing active and inactive lever pressing during extinction or reinstatement. (e) Location of injector tips in the NAcore that were used for all microinjection studies. Numbers indicate distance from bregma based on the rats atlas of Paxinos and Franklin (1997). Figure Ia, b and d include all rat behavioral data and histology, Figure Ic and d pool the reinstatement of all the saline-treated animals after FRI or FRI/FR3/FR5 schedules of reinforcement.

cocaine seeking (Smith et al, 2017)) in combination with LY 341495. Antagonizing nNOS failed to reduce the facilitation of reinstated sucrose seeking elicited by mGluR2/3 blockade (Figure 3a). Similarly, NPLA did not alter sucrose seeking that was not potentiated by simultaneous mGluR2/3 blockade.

\section{DREADD Activation of nNOS Potentiates Sucrose Seeking in Transgenic Mice}

mGluR5 is coupled to Gq signaling (Schoepp and Conn, 1993), and stimulation of the receptor induces calcium release from intracellular stores that can in turn initiate calmodulin-dependent activation of nNOS (Hayashi et al, 1999). Accordingly, we endeavored to super-activate Gq signaling selectively in NAcore nNOS interneurons using viral transfection of floxed Gq-DREADD in NOS1-Cre mice. We previously found that this strategy produced Gq-dependent depolarization in nNOS interneurons after stimulating the transfected receptor with the Gq-DREADD ligand clozapine-N-oxide (CNO), and that $\mathrm{CNO}$ stimulates $\mathrm{NO}$ production in transfected nNOS-Cre mice (Smith et al, 2017). Immunohistochemistry for nNOS and Cre in NOS1Cre mice revealed complete overlap of labeling for Cre in the nucleus of nNOS producing neurons in the NAcore (Figure 4a), and transfections and cannula tips were largely localized to the NAcore (Figure 4b; Supplementary
Figure 1E). Mice trained on an FR-1 schedule to selfadminister sucrose pellets discriminated active from inactive nose pokes, and underwent extinction training (Figure 4c). After extinction training, only the highest dose of $\mathrm{CNO}$ administration (3 mg/kg, i.p.) induced sucrose seeking in the absence of sucrose-paired cues (Figure 4d), and potentiated cued-induced reinstatement of sucrose seeking (Figure 4e). The increase in active nose poking was not associated with an increase in inactive nose poking (Figure $4 \mathrm{~d}$ and e). We also conducted an experiment using nNOS1-Cre mice that were trained using lever pressing as an operand that resulted in a similar effect by the highest dose of $\mathrm{CNO}$ to induce sucrose seeking in extinguished animals and to potentiate cue-induced reinstatement of sucrose seeking (Supplementary Figure 1). However, in this study, inactive lever pressing was also potentiated, although mice continued to significantly discriminate between the active and inactive lever during CNO-induced reinstatement.

To further evaluate the possibility of nonspecific behavioral activation we measured locomotor activity following $\mathrm{CNO}$ administration in animals habituated to an open field (Figure 4f). Mice showed a dose-dependent increase in locomotion after CNO administration, which became significant only at the highest dose of CNO. Although an overall increase in open field locomotion and active nose poking was induced by the same dose of CNO (3 mg/kg, i.p.), there was no correlation between $\mathrm{CNO}$-treated cue-induced 
reinstatement of sucrose seeking and open field locomotion (Figure 4g); suggesting that $\mathrm{CNO}$-induced increase in sucrose seeking is not entirely dependent on $\mathrm{CNO}$-induced
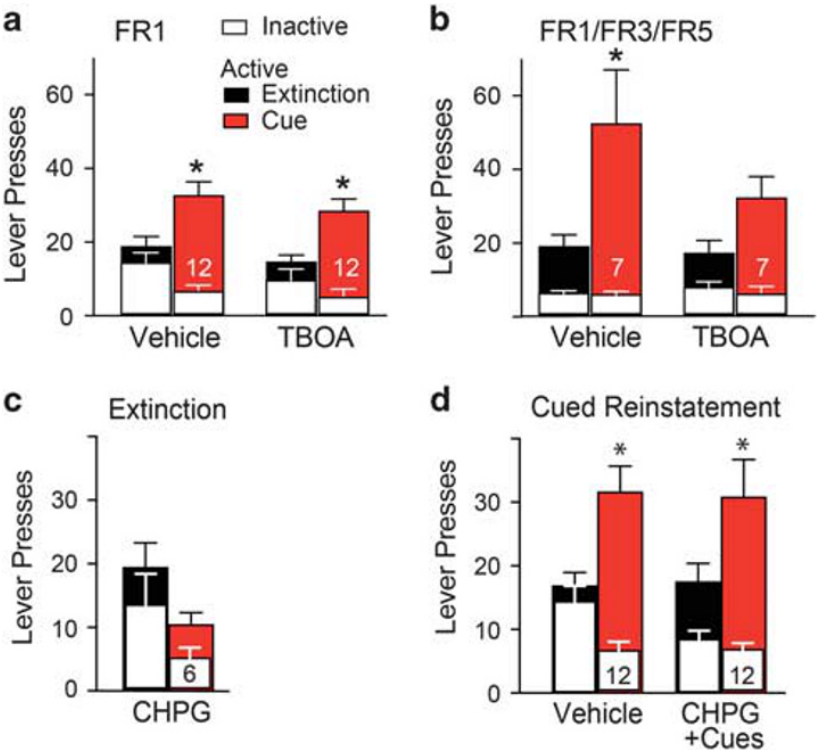

Figure 2 Blocking GLT-I uptake with TBOA before cued-reinstatement did not augment sucrose seeking. (a) Active and inactive lever presses after an FRI schedule of reinforcement and extinction training. Microinjections of vehicle or TBOA $(0.25 \mathrm{nmol} / 0.5 \mu \mathrm{l})$ were made $10 \mathrm{~min}$ before cuedreinstatement. The data were analyzed using both a three-way ANOVA (lever $\times$ treatment $\times$ extinction/cue), and using two separate two-way ANOVAs that separate the active from inactive levers. Three-way ANOVA revealed no effect of treatment $F_{(1,1)}=1.87, p=0.178$, an effect of active vs inactive lever $F_{(I, 1)}=31.14, p<0.0001$, an effect of extinction vs reinstatement $F_{(1,1)}=7.6, p=0.008$, and no interaction between treatment $\times$ lever or treatment $\times$ extinction/reinstatement. The two-way ANOVA for active lever revealed no interaction $F_{(1,22)}=8.618 \mathrm{e}-005, p=0.993$, no effect of treatment $F_{(1,22)}=1.50, p=0.234$, but an effect of extinction/reinstatement factor $F_{(1,22)}=26.32, p<0.001$. Inactive lever two-way ANOVA revealed no interaction $F_{(1,22)}=0.65, p=0.429$, no effect of treatment $F_{(1,22)}=2.09$, $p=0.162$, but a significant effect of extinction/reinstatement factor $F_{(1,22)}=9.73, p=0.005 . * p<0.05$ comparing extinction to reinstatement within each treatment for active or inactive levers using a Sidak's multiple comparisons post-hoc test. (b) Active and inactive lever presses after an FRI/ FR3/FR5 schedule of reinforcement and extinction training. Two-way ANOVA for active lever revealed no interaction $F_{(1,13)}=1.621, p=0.2252$, no effect of treatment $F_{(1,13)}=1.61, p=0.226$, but an effect of extinction/ reinstatement factor $F_{(1,13)}=11.25, p=0.005$. There is no significant effect in inactive lever pressing. $* 0<0.05$ comparing extinction to reinstatement within each treatment for active lever using a Sidak's multiple comparisons post-hoc test. $* 0<0.05$ comparing active lever presses between extinction and reinstatement within each treatment, using a Sidak's multiple comparisons post-hoc test. Each animal received a maximum of three reinstatement trials in a randomized crossover design. (c) No effect CHPG $(30 \mathrm{\mu g} / \mathrm{\mu l})$ microinjection into NAcore $10 \mathrm{~min}$ before an extinction session in rats trained to self-administer sucrose using an FRI schedule of reinforcement, two-way ANOVA revealed no effect of treatment $F_{(1.5)}=4.65, p=0.084$, active/inactive lever $F_{(1.5)}=3.19|, p=0.134|$, or overall interaction $F_{(1,5)}=0.01, p=0.913$. (d) Lack of effect by CHPG $(30 \mathrm{\mu g} / \mathrm{\mu l})$ microinjected into NAcore 10 min before a cued-reinstatement session in rats trained to self-administer sucrose using an FRI schedule of reinforcement, three-way ANOVA revealed no effect of treatment $F_{(I .1)}=0.89, p=0.347$, an effect of active/inactive lever $F_{(1.1)}=54.49$, $p<0.00 \mathrm{I}$, and an interaction between active/inactive lever and extinction/ reinstatement factors $F_{(I, 1)}=17.46, p<0.001$. The comparison between extinction and reinstatement was nearly significant $F_{(I, I)}=3.92, p=0.051$. $* p<0.05$ comparing active lever presses between extinction and reinstatement within each treatment using a Sidak's multiple comparisons post-hoc test. increase in locomotion. All experiments (extinction test, cued-reinstatement, and locomotion) were performed within-subjects.

\section{DISCUSSION}

Cues associated with drug delivery reinstate drug seeking following extinction training. Reinstated drug seeking depends on synaptic glutamate spillover in NAcore due to downregulated GLT-1 (Scofield et al, 2016a), and reduced negative regulation of presynaptic release probability by mGluR2/3 (Mulholland et al, 2016). We show that promoting glutamate spillover by blocking GLT-1 did not alter cued sucrose seeking, but that increasing release probability with an mGluR2/3 antagonist potentiated reinstated sucrose seeking. The facilitation of cue-induced sucrose seeking by blocking mGluR2/3 required stimulation of mGluR5, but in contrast to cocaine seeking, did not involve mGluR5 activation of nNOS (Smith et al, 2017). Nonetheless, akin to cocaine seeking directly stimulating nNOS activity using a chemogenetic Gq-DREADD strategy in nNOS-Cre mice potentiated sucrose seeking; although, this was associated with locomotor activation. Together, these data indicate that dysregulated presynaptic mGluR2/3 signaling is a possible site of shared signaling in reinstated drug seeking and potentiated sucrose seeking, but that downregulated glutamate transport and subsequent activation of nNOS is not shared.

\section{Glutamate Spillover and Cue-Induced Reinstatement}

Glutamate spillover into the extracellular space in the NAcore is necessary for cue-induced reinstatement of drug seeking (Scofield et al, 2016a). After withdrawal from all addictive drugs examined to date, the capacity of astroglia to remove glutamate is reduced due to decreased expression of the glutamate transporter GLT-1 and/or reduced proximity of astroglia to the synaptic cleft following repeated exposure to cocaine, alcohol, nicotine, or heroin (Mulholland et al, 2016; Scofield et al, 2016b). Also, after using cocaine, ethanol, or heroin, the capacity of mGluR2/3 to inhibit glutamate release probability is diminished due to receptor downregulation and/or increased expression of AGS3 that decreases Gi $\alpha$ signaling (Mulholland et al, 2016). Moreover, decreased basal glutamatergic levels after cocaine selfadministration further potentiate glutamate release by eliminating inhibitory tone on mGluR2/3 (Baker et al, 2003; Moran et al, 2005). In light of the abundant literature indicating that downregulated glutamate uptake is a common consequence of repeated use of many classes of addictive drug and that restoring the astroglial glutamate transporter inhibits cued drug seeking (Scofield et al, 2016a), we were surprised that enhancing extracellular glutamate concentrations by inhibiting GLT-1 with the antagonist TBOA did not potentiate reinstated sucrose seeking. Equally surprising was the distinction between the hypothesized glutamate spillover produced by blocking glutamate uptake and by microinjecting the mGluR2/3 antagonist LY 341495. This distinction likely arises from the fact that while mGluR2/3 blockade amplifies synaptic glutamate release associated with the sucrose conditioned cue, inhibiting 

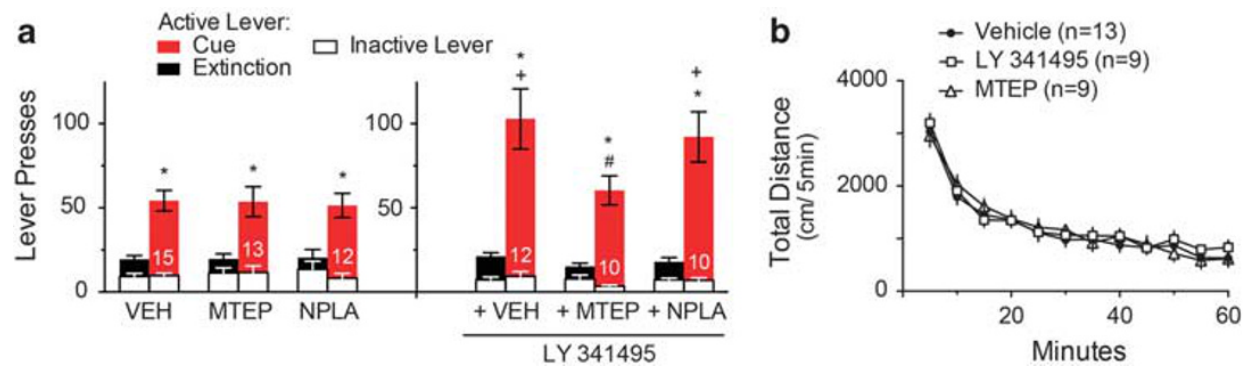

Figure 3 Blocking mGluR2/3 in NAcore before cued-reinstatement potentiated sucrose seeking compared to controls, by an mGluR5-dependent mechanism. (a) Rats were trained using the FRI/FR3/FR5 schedule (Figure Ib). All drugs were administered into NAcore 10 min prior to initiating cued

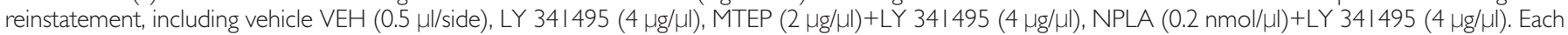
animal received a maximum of three reinstatement trials in a randomized crossover design. Two-way ANOVA for active lever, treatment $F_{(5,66)}=3.5 \mathrm{I}$, $p=0.007$, extinction/reinstatement $F_{(1,66)}=131.6, p<0.001$, interaction $F_{(5,66)}=4.309, p=0.0019$. There is no significant effect in inactive lever pressing. ${ }^{*} p<0.05$ comparing extinction to reinstatement within each treatment using a Sidak's multiple comparisons post-hoc test, ${ }^{+} p<0.05$ comparing active lever pressing to vehicle treatment group. ${ }^{*} p<0.05$ comparing active lever pressing of vehicle+LY34I 495 treatment group. (b) LY 34 I 495 (4 Hg/ $\left.\mu l\right)$ or MTEP (2 Hg/ Hl) microinjections into NAcore did not alter spontaneous locomotor activity. Data shown as mean \pm sem distance travelled a novel open-field. Two-way ANOVA with repeated measures over time, treatment $F_{(2,28)}=0.16, p=0.850$, time $F_{(11,308)}=98.22, p<0.001$, interaction $F_{(22,308)}=0.63, p=0.902$.

GLT-1 increases both synaptic and glial glutamate signaling in a manner that is unrelated to the specific circuit or engram activated by cue presentation (Carelli et al, 2000). Thus, while mGluR2/3 blockade amplifies glutamate release within a set of cortico-accumbens synapses encoding the association between cue and sucrose delivery, GLT-1 blockade enhances non-synaptic glutamatergic receptor signaling at synapses within and outside the engram coding the association. Thus, GLT-1 blockade may decrease the sucrose-seeking signal to noise ratio, which could account for why TBOA produced a trend towards reduced reinstated sucrose seeking on the more demanding FR schedule. This interpretation is consistent with the fact that the increase in basal extracellular glutamate produced by TBOA results almost entirely from the activity of the cystine-glutamate transporter, not synaptic activity (Baker et al, 2002; Bridges et al, 2012), and it is only when prefrontal to accumbens synaptic activity is increased during reinstated drug seeking that the elevated extracellular glutamate depends on synaptically released transmitter (LaLumiere and Kalivas, 2008; McFarland et al, 2003). It is also important to consider that the working doses of TBOA, CHPG, NPLA, and/or MTEP used in this study were based largely on doses that were effective in animals trained to selfadminister cocaine or other drugs of abuse (Griffin et al, 2014; Smith et al, 2017; Wang et al, 2013), and that chronic exposure to addictive drugs may have altered the Vmax or affinity of ligand binding. Indeed, cocaine produces constitutive reduction in GLT-1 (TBOA receptor (Knackstedt et al, 2010a)) and mGluR2/3 signaling (LY 341495 receptor (Scofield et al, 2016a)), and dephosphorylates nNOS (NPLA receptor (Rameau et al, 2004)), making it more responsive to calcium-induced activation. There are also studies showing drug-induced alterations in mGluR5 density and signaling (MTEP and CHPG receptor (Knackstedt et al, 2010b; Olive, 2009)). While this concern is not as relevant for drugs showing behavioral impact in this study (eg LY 341495 and MTEP), drugs not effective in modulating sucrose reinstatement are more concerning. The dose of NPLA used in this study is 10-fold higher than a dose effective in blocking cocaine reinstatement (Smith et al, 2017). As well, blockade at higher doses of TBOA or elimination of GLT-1 induces seizures (Rothstein et al, 1996), and we chose the dose of TBOA based on doses of TBOA in the nucleus accumbens (Griffin et al, 2014) or in other regions (Montiel et al, 2005) that increase extracellular glutamate levels.

While considering how different food diets and/or food restriction can impact glutamate homeostasis, it is important to highlight the impact of food regimens on post-synaptic glutamatergic receptors. Exposure to sucrose solutions (Peng et al, 2011; Tukey et al, 2013), highly unbalanced diet (Oginsky et al, 2016a), or food-restricted diet (Carr, 2016; Peng et al, 2011) increases AMPA receptor expression and changes its subunit composition in the nucleus accumbens. Moreover, the AMPA/NMDA ratio decreases after incubation of sucrose craving in adult mice trained to selfadminister sucrose (Counotte et al, 2014). These changes likely play a role in the regulation of synaptic strength, and hence on seeking behaviors.

\section{Subpopulations of mGluR5 Regulating Reinstatement}

In order for distinctions in the source of glutamate in the extracellular space to be important there may be different populations of extrasynaptic glutamate receptors that are accessed by the glial $v s$ neuronal sources of glutamate. Our findings indicate that inhibiting mGluR2/3 allows glutamate to access mGluR5 likely by inducing synaptic spillover, as a prerequisite for potentiating cue-reinstated sucrose seeking. The capacity of an mGluR5 NAM to prevent potentiated sucrose seeking was particularly intriguing since pharmacologically stimulating mGluR5 with CHPG did not alter reinstated sucrose seeking, in spite of the fact that previous studies found mGluR5 stimulation potentiates cocaine seeking (Schmidt et al, 2013; Smith et al, 2017; Wang et al, 2013). However, the capacity of CHPG to induce and potentiate cocaine reinstatement is probably enabled by the constitutive dephosphorylation of nNOS ser-847 after cocaine exposure (Smith et al, 2017), which facilitates the activation of nNOS by calmodulin (Rameau et al, 2004). The lack of effect of CHPG microinjection in the NAcore on reinstatement indicates not only that a specific subpopulation of mGluR5 is mediating potentiated sucrose 
a
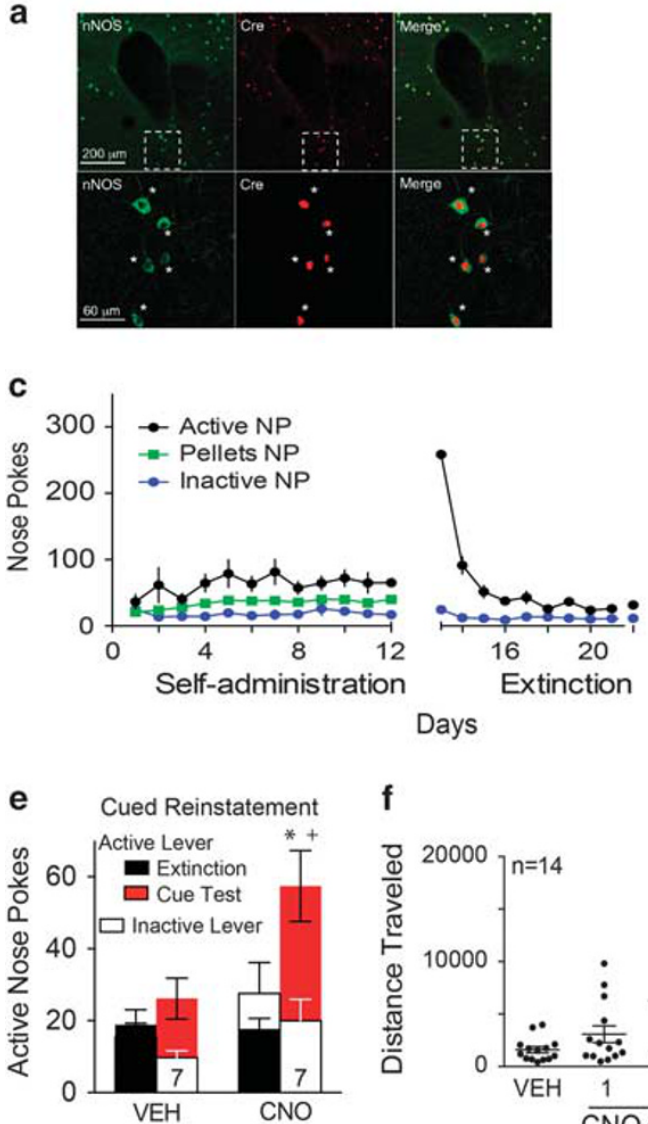

b

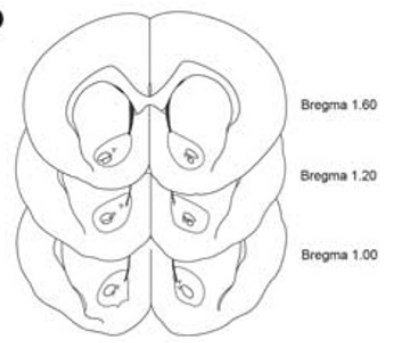

d

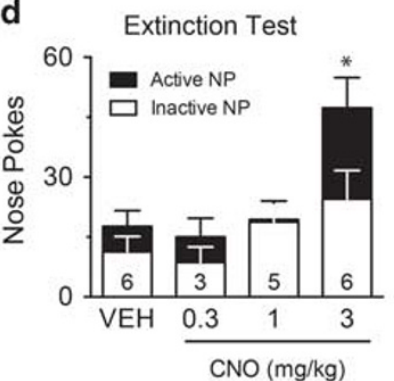

g
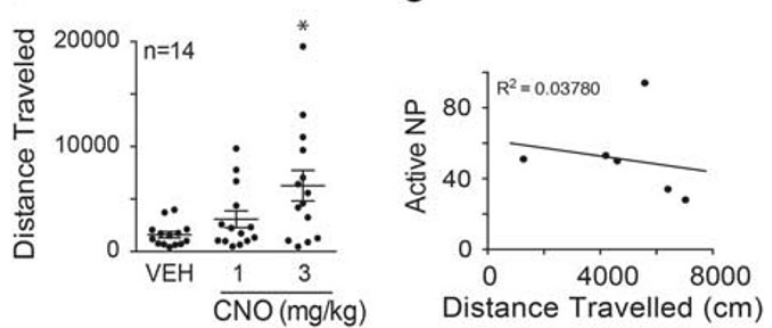

Figure 4 Gq-DREADD activation of NAcore nNOS interneurons potentiates reinstated sucrose seeking. (a) Cre expression is limited to nNOS interneurons. Immunohistochemistry in the NAcore for anti-nNOS (green) and anti-Cre (red) antibodies in NOSI-Cre mice. Asterisks indicate double-labeled Cre+/nNOS+ neurons. (b) Needle tip placements of Gq-DREADD virus injections in NOSI-Cre mice (trained with nose pokes) aimed at the NAcore. Numbers indicate distance from bregma based on the mouse atlas of Paxinos and Franklin (1997). (c) Mice were trained and extinguished from sucrose selfadministration on nose-pokes (NP) using an FRI schedule, $n=7$. (d) Dose-response curve of the effect of CNO-induced activation of Gq-DREADD transfected nNOS cells in the NAcore. CNO treatment increased active nose poking selectively in the absence of cues only with the highest concentration, $3 \mathrm{mg} / \mathrm{kg}$. We performed paired $t$-tests with Bonferroni correction for multiple comparisons between the vehicle group (VEH) and each CNO dose for active and inactive nose pokes. Only the $3 \mathrm{mg} / \mathrm{kg} \mathrm{CNO}$ group was statistically different to the VEH for active nose pokes, * $p=0.0 \mathrm{Il}$. Each animal received a minimum of one vehicle and two CNO doses. (e) Activation of nNOS cells during cued-reinstatement by the highest dose of CNO (3 mg/kg) increases active nose poking. Two-way ANOVA for active nose poke revealed no significant effect of treatment $F_{(1,12)}=4.09, p=0.066$, but a significant effect of extinction vs cued-reinstatement test $F_{(1,12)}=25.38, p<0.001$ and a significant interaction $F_{(1,12)}=12.08, p=0.005$. $* 0<0.05$ comparing active nose pokes between extinction and reinstatement within each treatment, $+p<0.05$ comparing active nose pokes during cued-reinstatement within each treatment. Two-way ANOVA for inactive nose poke revealed no significant effect of treatment $F_{(1,12)}=2.40, p=0.148$, extinction vs cued-reinstatement test $F_{(1,12)}=4.11$, $p=0.066$ or interaction $F_{(1,12)}=0.067, p=0.800$. Each mouse received a maximum of two extinction tests and reinstatement trials in a randomized crossover design. (f) CNO-induced locomotion in an open-field, mice received vehicle (Veh) or different doses of CNO (I mg/kg and $3 \mathrm{mg} / \mathrm{kg}$ ) $30 \mathrm{~min}$ before being placed into a pre-habituated open-field. Randomized crossover design, $n=14$ (includes mice self-administering sucrose with levers with levers and nose pokes), repeated measures, one-way ANOVA, $F_{(1.398,18.18)}=8.78, p=0.005$, $* p<0.05$ comparing VEH to CNO 3 mg/kg. (g) Lack of correlation between the $3 \mathrm{mg} / \mathrm{kg} \mathrm{CNO}$-induced active nose poking during cued-reinstatement test and the $3 \mathrm{mg} / \mathrm{kg} \mathrm{CNO}$-induced open field locomotion ( $2 \mathrm{~h}$ for both tests), linear regression $\mathrm{F}_{(1,4)}=0.16, p=0.7120, R^{2}=0.0378$

reinstatement, but also that simultaneous pharmacological activation of all mGluR5 by CHPG may activate multiple populations of mGluR5 that produce countermanding effects on reinstated sucrose seeking.

mGluR5 are located on most neurons (Tallaksen-Greene et al, 1998) and on astrocytes (Porter and McCarthy, 1997), although glial expression of mGluR5 remains controversial (Sun et al, 2013). mGluR5 located on nNOS-expressing interneurons is critical for the manifestation of reinstated cocaine seeking (Smith et al, 2017). However, this population was not required for mGluR2/3-potentiated sucrose seeking since blocking nNOS with NPLA had no effect on reinstated sucrose seeking. While we did not directly test other cellspecific populations of mGluR5, it is interesting that mGluR5 inhibition reduced only mGluR $2 / 3$ potentiated sucrose seeking, and not baseline cue-induced sucrose seeking (Figure 3a (Sinclair et al, 2012)) or sweetened food reward (Tronci et al, 2010). This indicates that the critical population of mGluR5 is selectively accessed by synaptic glutamate spillover that arises from inhibiting presynaptic mGluR2/3. The most likely population in this regard is mGluR5 expressed postsynaptically on NAcore Medium Spiny Neurons (MSNs) (Tallaksen-Greene et al, 1998). However, a well-characterized effect of stimulating this 
mGluR5 population is inducing an endocannabinoiddependent long-term depression (LTD) (Grueter et al, 2008; Huang et al, 2011; Kreitzer and Malenka, 2005). Endocannabinoid-mediated LTD arises from stimulating presynaptic CB1 receptors that, akin to $\mathrm{mGluR} 2 / 3$, are Gi $\alpha$ coupled and inhibit glutamate release probability (Robbe et al, 2001). Thus, antagonizing this population of mGluR5 would be expected to promote, not reduce the potentiated reinstatement elicited by blocking mGluR2/3. Another potential population to consider is mGluR5 expression on astroglia. Stimulating this population of mGluR5 is hypothesized to promote calcium-dependent release of glutamate from astroglia, which stimulates GluN2B-expressing NMDA channels on MSNs (D'Ascenzo et al, 2007). Thus, by indirectly promoting NMDA receptor activation on MSNs, stimulation of astroglial mGluR5 could potentiate glutamatergic synaptic input to MSNs and potentiate reinstated sucrose seeking.

\section{nNOS Interneuron Regulation of Reinstated Sucrose Seeking}

We employed a Gq-DREADD strategy to promote Gq signaling selectively in nNOS-expressing NAcore interneurons. Given the negative findings with the mGluR5 agonist CHPG, it was surprising that stimulating Gq-DREADD in the nNOS interneurons potentiated cue-reinstated sucrose seeking, as was previously shown for reinstated cocaine seeking (Smith et al, 2017). Increased reinstatement was associated with an increase in locomotor activity, although locomotion was not correlated with reinstated sucrose seeking. CNO had no significant effect on inactive nose poking. The disconnection between stimulating endogenous Gq-coupled mGluR5 with CHPG and transfected Gq-DREADD may arise from the overexpression of the DREADD producing non-physiological activation of nNOS. This is indicated by the fact that cocaine self-administration produces an enduring reduction in nNOS phosphorylation that facilitates enzyme activation through mGluR5 signaling, and likely accounts for CHPG potentiation of cue-induced cocaine seeking (Smith et al, 2017). Alternatively, simultaneous stimulation of all mGluR5 in NAcore by microinjected CHPG may produce countermanding effects on the capacity of conditioned cues to reinstate sucrose seeking. For example, while CHPG stimulation of nNOS interneurons bearing mGluR5 may promote reinstated sucrose seeking, simultaneous stimulation of mGluR5 on MSNs could elicit countermanding LTD (Grueter et al, 2008; Huang et al, 2011; Kreitzer and Malenka, 2005). In contrast, the balance between mGluR5 on nNOS interneurons and MSNs may be altered in favor of nNOS in cocaine-extinguished animals by the constitutive dephosphorylation of nNOS.

\section{Conclusions}

We show that increasing glutamate release by blocking mGluR2/3 in the NAcore before cue-induced sucrose reinstatement stimulated a subpopulation of mGluR5 and potentiated sucrose seeking. In contrast, increasing predominantly glial glutamate spillover by blocking GLT-1 did not alter reinstated sucrose seeking. Since cocaine and other addictive drugs produce cue-induced glutamate spillover by both a presynaptic mGluR2/3 and a GLT-1 mechanism, these data argue that seeking addictive drugs involves both overlapping and distinct cellular mechanisms with those activated by sucrose seeking. Moreover, the data indicate that while endogenous impairments in glial regulation of extracellular glutamate are not likely to contribute, impairments regulating presynaptic cortico-accumbens glutamate release could predispose individuals to eating disorders.

\section{FUNDING AND DISCLOSURE}

This work was supported by a post-doctoral fellowship from the French Fyssen Foundation (ACB) and the National Institute of Health NIH DA003906 and DA12513 (PWK). The authors have nothing to disclose.

\section{ACKNOWLEDGMENTS}

The authors would like to thank Dr Louk Vanderschuren and Dr Kate Reissner for helpful discussions, Dr Angie M. Cason for useful comments on rat sucrose self-administration, Dr Heather A. Boger for allowing us to perform the rat locomotor measurement using her equipment, Dr William C. Griffin III for allowing the use of the mice locomotor boxes, as well as Meghan Grandal for her help with the nNOS mouse behavior.

\section{REFERENCES}

Ahmed SH, Guillem K, Vandaele Y (2013). Sugar addiction: pushing the drug-sugar analogy to the limit. Curr opin clin nutr metab care 16: 434-439.

Baker DA, McFarland K, Lake RW, Shen H, Tang XC, Toda S et al (2003). Neuroadaptations in cystine-glutamate exchange underlie cocaine relapse. Nat Neurosci 6: 743-749.

Baker DA, Xi ZX, Shen H, Swanson CJ, Kalivas PW (2002). The origin and neuronal function of in vivo nonsynaptic glutamate. J neurosci 22: 9134-9141.

Baptista MA, Martin-Fardon R, Weiss F (2004). Preferential effects of the metabotropic glutamate $2 / 3$ receptor agonist LY379268 on conditioned reinstatement versus primary reinforcement: comparison between cocaine and a potent conventional reinforcer. J neurosci 24: 4723-4727.

Bechara A (2005). Decision making, impulse control and loss of willpower to resist drugs: a neurocognitive perspective. Nat Neurosci 8: 1458-1463.

Bossert JM, Gray SM, Lu L, Shaham Y (2006). Activation of group II metabotropic glutamate receptors in the nucleus accumbens shell attenuates context-induced relapse to heroin seeking. Neuropsychopharmacology 31: 2197-2209.

Bridges R, Lutgen V, Lobner D, Baker DA (2012). Thinking outside the cleft to understand synaptic activity: contribution of the cystine-glutamate antiporter (System $\mathrm{xc}$-) to normal and pathological glutamatergic signaling. Pharmacol Rev 64: 780-802.

Brown RM, Kupchik YM, Spencer S, Garcia-Keller C, Spanswick DC, Lawrence AJ et al (2015). Addiction-like synaptic impairments in diet-induced obesity. Biol Psychiatry 81: 797-806.

Carelli RM, Ijames SG, Crumling AJ (2000). Evidence that separate neural circuits in the nucleus accumbens encode cocaine versus 'natural' (water and food) reward. J neurosci 20: $4255-4266$. 
Carr KD (2016). Nucleus accumbens AMPA receptor trafficking upregulated by food restriction: an unintended target for drugs of abuse and forbidden foods. Curr Opin Behav Sci 9: 32-39.

Counotte DS, Schiefer C, Shaham Y, O'Donnell P (2014). Timedependent decreases in nucleus accumbens AMPA/NMDA ratio and incubation of sucrose craving in adolescent and adult rats. Psychopharmacology (Berl) 231: 1675-1684.

D'Ascenzo M, Fellin T, Terunuma M, Revilla-Sanchez R, Meaney DF, Auberson YP et al (2007). mGluR5 stimulates gliotransmission in the nucleus accumbens. Proc Natl Acad Sci USA 104: 1995-2000.

Danbolt NC (2001). Glutamate uptake. Prog Neurobiol 65: $1-105$.

Griffin WC 3rd, Haun HL, Hazelbaker CL, Ramachandra VS, Becker HC (2014). Increased extracellular glutamate in the nucleus accumbens promotes excessive ethanol drinking in ethanol dependent mice. Neuropsychopharmacology 39: 707-717.

Grueter BA, McElligott ZA, Robison AJ, Mathews GC, Winder DG (2008). In vivo metabotropic glutamate receptor 5 (mGluR5) antagonism prevents cocaine-induced disruption of postsynaptically maintained mGluR5-dependent long-term depression. J Neurosci 28: 9261-9270.

Hayashi Y, Nishio M, Naito Y, Yokokura H, Nimura Y, Hidaka H et al (1999). Regulation of neuronal nitric-oxide synthase by calmodulin kinases. J Biol Chem 274: 20597-20602.

Huang CC, Yeh CM, Wu MY, Chang AY, Chan JY, Chan SH et al (2011). Cocaine withdrawal impairs metabotropic glutamate receptor-dependent long-term depression in the nucleus accumbens. J neurosci 31: 4194-4203.

Knackstedt LA, Melendez RI, Kalivas PW (2010a). Ceftriaxone restores glutamate homeostasis and prevents relapse to cocaine seeking. Biol Psychiatry 67: 81-84.

Knackstedt LA, Moussawi K, Lalumiere R, Schwendt M, Klugmann M, Kalivas PW (2010b). Extinction training after cocaine self-administration induces glutamatergic plasticity to inhibit cocaine seeking. J neurosci 30: 7984-7992.

Kreitzer AC, Malenka RC (2005). Dopamine modulation of state-dependent endocannabinoid release and long-term depression in the striatum. J Neurosci 25: 10537-10545.

LaLumiere RT, Kalivas PW (2008). Glutamate release in the nucleus accumbens core is necessary for heroin seeking. $J$ neurosci 28: $3170-3177$.

Luscher C, Huber KM (2009). Group 1 mGluR-dependent synaptic long-term depression: mechanisms and implications for circuitry and disease. Neuron 65: 445-459.

Luscher C, Malenka RC (2011). Drug-evoked synaptic plasticity in addiction: from molecular changes to circuit remodeling. Neuron 69: 650-663.

McFarland K, Lapish CC, Kalivas PW (2003). Prefrontal glutamate release into the core of the nucleus accumbens mediates cocaineinduced reinstatement of drug-seeking behavior. J neurosci 23: 3531-3537.

Montiel T, Camacho A, Estrada-Sanchez AM, Massieu L (2005). Differential effects of the substrate inhibitor l-trans-pyrrolidine2,4-dicarboxylate (PDC) and the non-substrate inhibitor DL-threo-beta-benzyloxyaspartate (DL-TBOA) of glutamate transporters on neuronal damage and extracellular amino acid levels in rat brain in vivo. Neuroscience 133: 667-678.

Moran MM, McFarland K, Melendez RI, Kalivas PW, Seamans JK (2005). Cystine/glutamate exchange regulates metabotropic glutamate receptor presynaptic inhibition of excitatory transmission and vulnerability to cocaine seeking. $J$ neurosci 25: 6389-6393.

Moussawi K, Kalivas PW (2010). Group II metabotropic glutamate receptors $(\mathrm{mGlu}(2 / 3))$ in drug addiction. Eur J Pharmacol 639: $115-122$.
Moussawi K, Zhou W, Shen H, Reichel CM, See RE, Carr DB et al (2011). Reversing cocaine-induced synaptic potentiation provides enduring protection from relapse. Proc Natl Acad Sci USA 108: 385-390.

Mulholland PJ, Chandler LJ, Kalivas PW (2016). Signals from the fourth dimension regulate drug relapse. Trends neurosci 39: 472-485.

Oginsky MF, Goforth PB, Nobile CW, Lopez-Santiago LF, Ferrario CR (2016a). Eating 'junk-food' produces rapid and long-lasting increases in NAc CP-AMPA receptors: implications for enhanced cue-induced motivation and food addiction. Neuropsychopharmacology 41: 2977-2986.

Oginsky MF, Maust JD, Corthell JT, Ferrario CR (2016b). Enhanced cocaine-induced locomotor sensitization and intrinsic excitability of NAc medium spiny neurons in adult but not in adolescent rats susceptible to diet-induced obesity. Psychopharmacology (Berl) 233: 773-784.

Oldenziel WH, Dijkstra G, Cremers TI, Westerink BH (2006). In vivo monitoring of extracellular glutamate in the brain with a microsensor. Brain Res 1118: 34-42.

Olive MF (2009). Metabotropic glutamate receptor ligands as potential therapeutics for addiction. Curr Drug Abuse Rev 2: 83-98.

Paxinos G, Franklin KBJ. The Mouse Brain in Stereotaxic Coordinates, 2nd edn. Academic Press: New York, 1997.

Peng XX, Ziff EB, Carr KD (2011). Effects of food restriction and sucrose intake on synaptic delivery of AMPA receptors in nucleus accumbens. Synapse 65: 1024-1031.

Peters J, Kalivas PW (2006). The group II metabotropic glutamate receptor agonist, LY379268, inhibits both cocaine- and food-seeking behavior in rats. Psychopharmacology (Berl) 186: 143-149.

Porter JT, McCarthy KD (1997). Astrocytic neurotransmitter receptors in situ and in vivo. Prog Neurobiol 51: 439-455.

Rameau GA, Chiu LY, Ziff EB (2004). Bidirectional regulation of neuronal nitric-oxide synthase phosphorylation at serine 847 by the $N$-methyl-D-aspartate receptor. J Biol Chem 279: 14307-14314.

Rao PS, Sari Y (2012). Glutamate transporter 1: target for the treatment of alcohol dependence. Curr med chem 19: 5148-5156.

Robbe D, Alonso G, Duchamp F, Bockaert J, Manzoni OJ (2001). Localization and mechanisms of action of cannabinoid receptors at the glutamatergic synapses of the mouse nucleus accumbens. J Neurosci 21: 109-116.

Rothstein JD, Dykes-Hoberg M, Pardo CA, Bristol LA, Jin L, Kunci RW et al (1996). Knockout of glutamate transporters reveals a major role for astroglial transport in excitotoxicity and clearance of glutamate. Neuron 16: 675-686.

Schmidt HD, Schassburger RL, Guercio LA, Pierce RC (2013). Stimulation of mGluR5 in the accumbens shell promotes cocaine seeking by activating PKC gamma. J Neurosci 33: 14160-14169.

Schoepp D, Conn P (1993). Metabotropic glutamate receptors in brain function and pathology. TiPS 14: 13-20.

Schreiber LR, Odlaug BL, Grant JE (2013). The overlap between binge eating disorder and substance use disorders: diagnosis and neurobiology. J behav addict 2: 191-198.

Schultz W (2011). Potential vulnerabilities of neuronal reward, risk, and decision mechanisms to addictive drugs. Neuron 69: 603-617.

Scofield MD, Heinsbroek JA, Gipson CD, Kupchik YM, Spencer S, Smith AC et al (2016a). The nucleus accumbens: mechanisms of addiction across drug classes reflect the importance of glutamate homeostasis. Pharmacol Rev 68: $816-871$

Scofield MD, Li H, Siemsen BM, Healey KL, Tran PK, Woronoff N et al (2016b). Cocaine self-administration and extinction leads to reduced glial fibrillary acidic protein expression and 
morphometric features of astrocytes in the nucleus accumbens core. Biol Psychiatry 80: 207-215.

Sinclair CM, Cleva RM, Hood LE, Olive MF, Gass JT (2012). mGluR5 receptors in the basolateral amygdala and nucleus accumbens regulate cue-induced reinstatement of ethanol-seeking behavior. Pharmacol biochem behav 101: 329-335.

Smith AC, Scofield MD, Heinsbroek JA, Gipson CD, Neuhofer D, Roberts-Wolfe DJ et al (2017). Accumbens nNOS interneurons regulate cocaine relapse. J neurosci 37: 742-756.

Sun W, McConnell E, Pare JF, Xu Q, Chen M, Peng W et al (2013). Glutamate-dependent neuroglial calcium signaling differs between young and adult brain. Science 339: 197-200.

Tallaksen-Greene SJ, Kaatz KW, Romano C, Albin RL (1998). Localization of mGluR1a-like immunoreactivity and mGluR5-like immunoreactivity in identified populations of striatal neurons. Brain Res 780: 210-217.
Tomasi D, Volkow ND (2013). Striatocortical pathway dysfunction in addiction and obesity: differences and similarities. Crit Rev Biochem Mol Biol 48: 1-19.

Tronci V, Vronskaya S, Montgomery N, Mura D, Balfour DJ (2010). The effects of the mGluR5 receptor antagonist 6-methyl-2(phenylethynyl)-pyridine (MPEP) on behavioural responses to nicotine. Psychopharmacology (Berl) 211: 33-42.

Tukey DS, Ferreira JM, Antoine SO, D'Amour JA, Ninan I, Cabeza de Vaca S et al (2013). Sucrose ingestion induces rapid AMPA receptor trafficking. I neurosci 33: 6123-6132.

Wang X, Moussawi K, Knackstedt L, Shen H, Kalivas PW (2013). Role of mGluR5 neurotransmission in reinstated cocaine-seeking. Addict Biol 18: 40-49.

Wolf ME (2010). The Bermuda Triangle of cocaine-induced neuroadaptations. Trends Neurosci 33: 391-398.

Supplementary Information accompanies the paper on the Neuropsychopharmacology website (http://www.nature.com/npp) 